\title{
An Approach for Fast Segmentation of Lung Cancer Images
}

\author{
A Saraiva ${ }^{1}$, MS Oliveira ${ }^{2 *}$, JVM Sousa ${ }^{1}$, NM Fonseca Ferreira ${ }^{3}$, Antonio Valente ${ }^{4,5}$ and \\ Salviano Soares ${ }^{4}$ \\ ${ }^{1}$ State University of Piaui, Brazil \\ ${ }^{2}$ Department of Computing and Mathematics, Brazil \\ ${ }^{3}$ Institute of Engineering of Coimbra, Portugal \\ ${ }^{4}$ UTAD University, Portugal \\ ${ }^{5}$ School of Science and Technology, UTAD University, Vila Real, Portugal
}

*Corresponding author: MS Oliveira, Department of Computing and Mathematics, University of São Paulo, Ribeirão Preto, Brazil

\section{ARTICLE INFO \\ Received: 絊 January 22, 2019 \\ Published: 㓞 February 07, 2019}

Citation: A Saraiva, MS Oliveira*, JVM Sousa, NM Fonseca Ferreira, Antonio Valente, et al., An Approach for Fast Segmentation of Lung Cancer Images. Biomed J Sci \& Tech Res 14(2)-2019. BJSTR. MS.ID.002510.

\author{
ABSTRACT
}

The lung tumor is a leading cause of death worldwide. This type of disease in which there is uncontrolled progression of abnormal cells in the lung. To quantify these carcinogenic structures in the lung, medical images such as Computed Tomography (CT) and Magnetic Resonance Imaging (MRI) are used, enabling preoperative planning and treatment, however, lung tumor segmentation is a challenging. The method illustrated in this review is based on the region growing techniques and the results obtained are motivating, finally, a statistical analysis and a graphic demonstration of the proposed algorithm are presented.

Keywords: Lung Cancer; Automatic Segmentation; 3D Slicer

\section{Introduction}

The lung tumor is a leading cause of death worldwide. This type of disease in which there is uncontrolled progression of abnormal cells in the lung, when untreated, can extend through the process of metastasis in nearby tissues and in different parts of the body [1]. According to the study discussed in [2], the majority of lung cancer cases occur through cigarette use. As a curative approach it is usually recommended to combine radiotherapy and chemotherapy, in other hands, the earlier the tumor identification the better the patient's survival rate $[2,3]$. To quantify these carcinogenic structures in the lung, medical images such as Computed Tomography (CT) and Magnetic Resonance Imaging (MRI) are used, enabling preoperative planning and treatment, however, lung tumor segmentation is a challenging. In order to solve the challenge of segmentation of images, several algorithms in the literature are illustrated, among them, there are methods that use adaptive threshold [4-8], fuzzy models [9-11], region growing [12-14]. Motivated by these studies the present review seeks to illustrate the development of a new combined method for fast segmentation.

\section{The Method}

The method illustrated in this review is initially based on the region growing techniques present in 3D Slicer [15]. Known as Grow Cut [16], this algorithm uses cellular automata to identify neighboring pixels and tag them with an ID equal to the seed points given at the beginning of the algorithm. For this, this method requires the marking of foreground and background seed. Usually the background seed should mark a closed outline around the target area (foreground seed), so the algorithm will run until all pixels that have similarity with target points are marked. However, noting that all pixels will be visited during each iteration, the complexity grows rapidly as a result of the increase of the image size, reducing its applicability for 3D image segmentation. In order to improve the Grow Cut results, a modification of its implementation was proposed in [17]. The algorithm called Fast Grow Cut solved the runtime challenge using a method based on graph algorithms more specifically known as Dijkstra, in which it is able to calculate the minimum cost path between the vertices of a graph, thus 
generating a good performance of execution. However, these two versions created for image segmentation sometimes result in parts of the segmented structures that generate rough structures when they are illustrated in 3D, in addition, these methods do not automatically generate 3D visualization at the end of its execution, forcing the user to do it manually later.

In our method, initially only seed foreground image (SF) is required. From this image, its marked target coordinates are extracted, these coordinates are stored and subsequently used as indices for extracting pixel values from the input image (I) to be segmented at the same point. Thus, two vectors are created, the first, corresponding to the target zone coordinates (TZC) and the second, containing the corresponding pixel values (CZV). Parallel to this, the image that will be used as output $(\mathrm{S})$ is created by cloning SF to ensure the same dimensions in the images. After this step, a range with the highest and lowest value is identified and created by applying histogram in CZV. When creating the initial vectors, as well as the cut range, the algorithm starts the iteration of region growing, checking at the beginning if it is already at the edge of the image (edges), if this occurs, the algorithm ends, otherwise the algorithm proceeds to capture an adjacent voxel (AV) in I, picks up its values, coordinates and enters a new test case. If the $A V$ values are within the range created initially, it will have its indices stored in a new vector (NV), but if there is no new voxel detected within the range then the algorithm is terminated.
On the other hand, if there is, the algorithm executes an interaction in $\mathrm{NV}$ taking its matches which are later used to capture the voxel in S by storing it in a local variable (LA). In this sense, after this step, the NV coordinates are used to obtain the value of the pixel in this string in I by storing it in (LV) and the voxel in I by storing it in (LB). After these two steps, a product is executed between LA and LB, then a local cut range is created, taking the lowest value and the largest of the result of the previous product, if $\mathrm{LV}$ is between this range, then $\mathrm{S}$ in the $\mathrm{NV}$ is marked, the pass to next iteration. At the end of the growing method, a border smoothing is performed. For this smoothing to be performed, it is iterated in all slices. Each slice is converted to binary image, then this slice goes through a binary threshold isolating the area of interest and its result is smoothed shortly after combining two techniques of mathematical morphology, opening and closing, respectively, with an elliptical structuring element size (5x5). After of this step the result is converted back to the initial image format and demonstrates through the modules available in the 3DSlicer, the $3 \mathrm{D}$ reconstruction of the segmentation results automatically.

\section{Current results}

The results obtained are motivating. In Table 1 the efficiency in relation to the execution time of the method is shown, taking into account only the time to segment the medical image. Note that the proposed method can reduce the execution time of such a task (Figures 1-3).

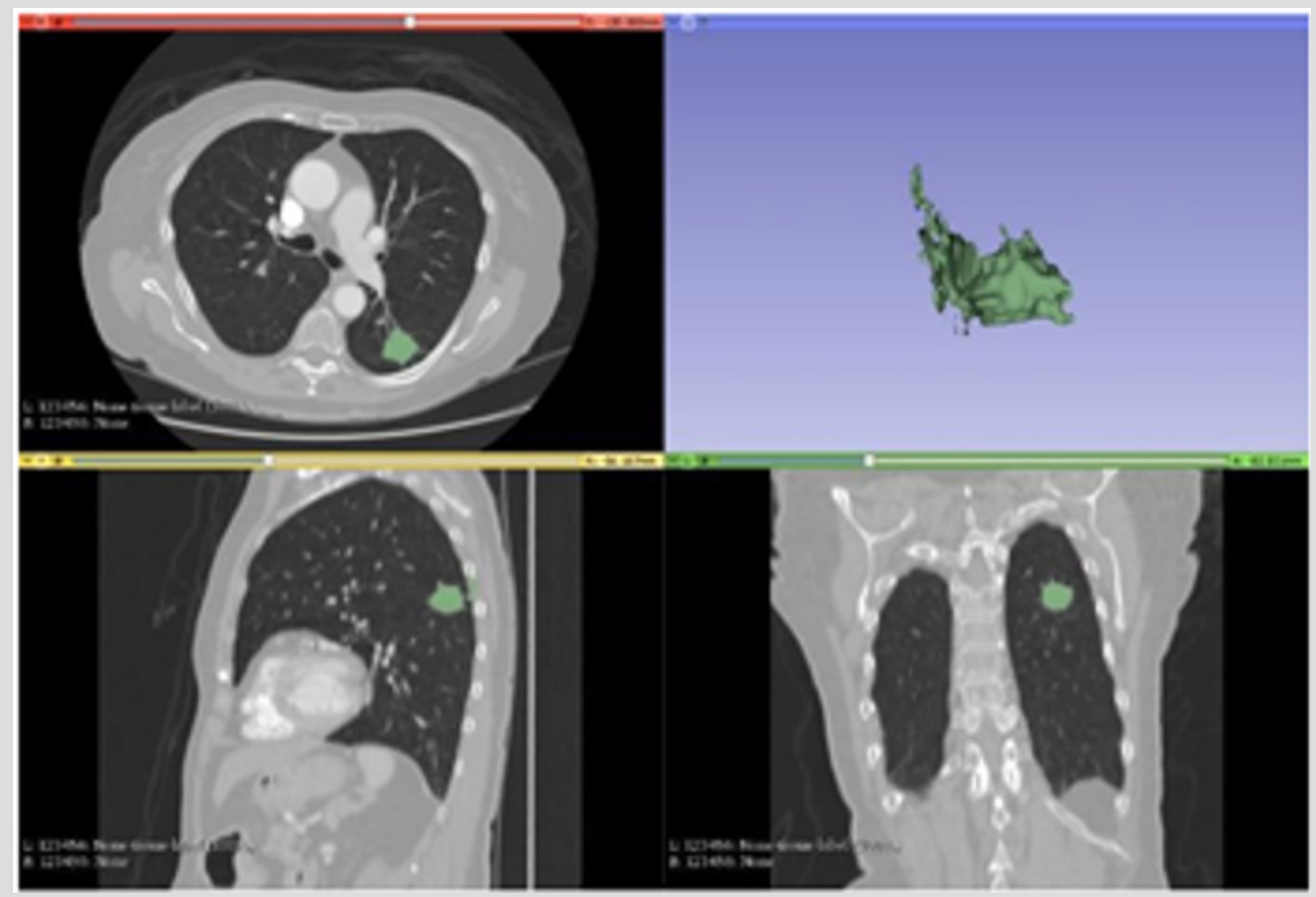

Figure 1: Segmentation with Grow cut.

Note: The Figure 1 demonstrates the segmentation result of the Grow Cut method. In addition to the runtime being the largest among the tests as illustrated in the previous table, its segmentation process captured many artifacts outside the target area expected in the test in question, generating a $3 \mathrm{~d}$ with a less accurate tumor structure. 


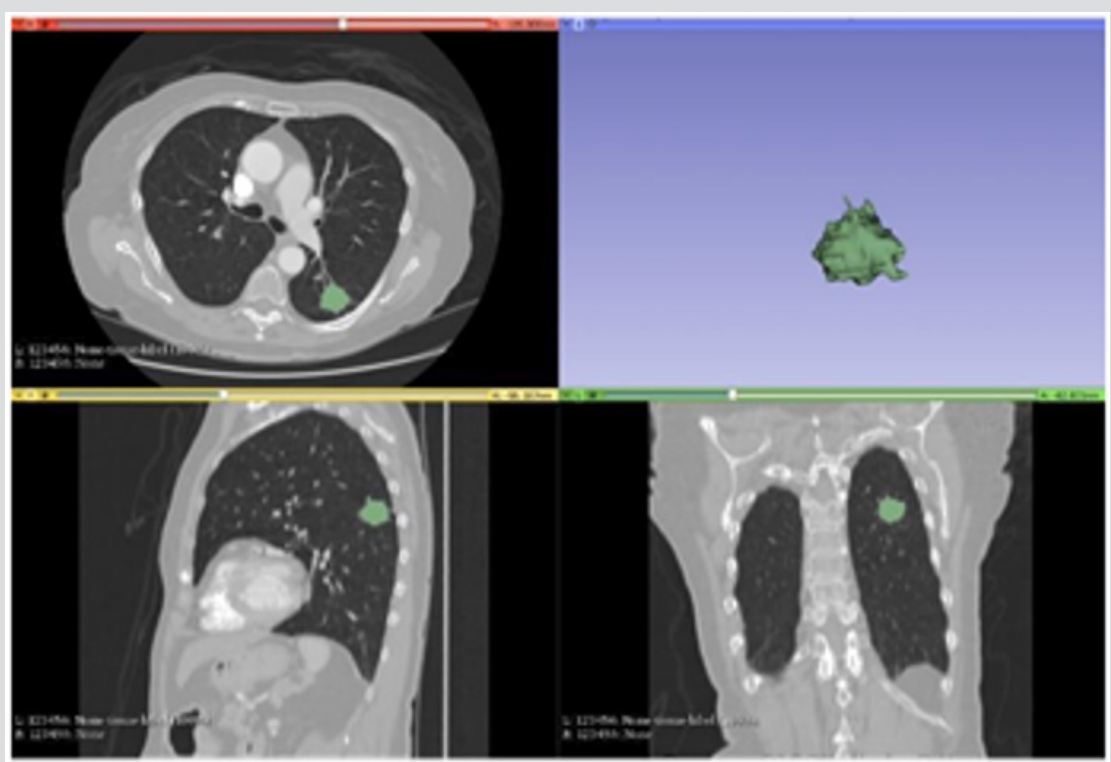

Figure 2: Segmentation with Fast Grow Cut.

Note: The Figure 2 demonstrates the segmentation result of the Fast Grow Cut method. This method was able to perform the task reducing the time required drastically and is shown with very few false positives, however it is necessary to manually generate by other modules the resulting $3 \mathrm{D}$.

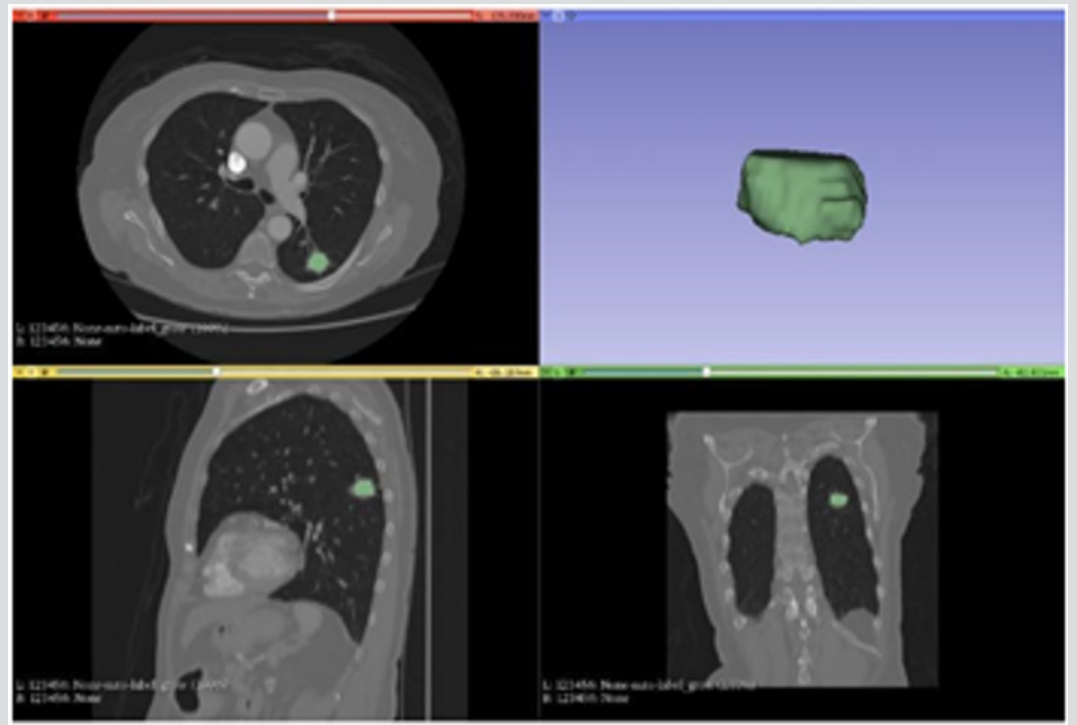

Figure 3: Segmentation with proposed method.

Note: The Figure 3 illustrates the result of the proposed algorithm. a particularity of this algorithm is given by the automation of the whole process, from the marking of the area of interest at the beginning all the remaining process until the 3D illustration is done automatically, in other hands the algorithm was also able to reduce the time for the execution of the segmentation task even more, staying in fast 2 s.

Table 1: Runtime for segmentation.

\begin{tabular}{|c|c|}
\hline Grow Cut & $41 \mathrm{~s}$ \\
\hline Fast Grow Cut & $6 \mathrm{~s}$ \\
\hline Proposed Method & $2 \mathrm{~s}$ \\
\hline
\end{tabular}

\section{Discussion}

With the introduction of the new method it is evident that it has potential for improvement in the resolution of the proposed task, making the result more quickly and with simple use. Compared to two other algorithms it has been shown to be faster in the segmentation process, it has automatic 3D visualization generation, but it is still feasible to improve in some sections.

\section{Conclusion}

There are several techniques for segmentation of medical images, this study seeks to demonstrate a new algorithm developed. The efficiency of the work developed is due to the automation of processes and the reduction in the cost of time for execution. The study of the method could generate more advances, generating a 
better performance for segmentation. The disadvantage is the loss of some structures due to the currently developed smoothing technique, in this sense, an optimization is proposed initially in this process. However, the process of smoothing is still very aggressive even removing structures beyond only noisy imperfections, it is proposed at the moment to improve this process. As additional information, but not little relevant to the project, the execution of the complete method until the 3D generation takes about $8 \mathrm{~s}$. The tests were performed on a Notebook with Intel Core i3 $2 \mathrm{GHz} 4 \mathrm{~GB}$ RAM.

\section{Acknowledgment}

This work is financed by the National Funds through the FCT Fundação para a Ciência e a Tecnologia (Portuguese Foundation for Science and Technology) as part of project UID/EEA/00760/2019.

\section{References}

1. Sangamithraa PB, Govindaraju S (2016) Lung tumour detection and classification using EK-Mean clustering. In Wireless Communications, Signal Processing and Networking (WiSPNET), International Conference on pp. 2201-2206.

2. Patel V, Talati B (2017) A Study of Image Segmentation and Classification of Lung Tumors. International Journal of Scientific Research in Science, Engineering and Technology pp. 360-365.

3. Curran Jr WJ, Paulus R, Langer CJ, Komaki R, Lee JS, et al. (2011) Sequential vs concurrent chemoradiation for stage III non-small cell lung cancer: randomized Phase III trial RTOG 9410. Journal of the National Cancer Institute 103(19): 1452-1460.

4. Erdi YE, Mawlawi O, Larson SM, Imbriaco M, Yeung H, et al. (1997) Segmentation of lung lesion volume by adaptive positron emission tomography image thresholding. Cancer 80(S12): 2505-2509.

5. Greco C, Rosenzweig K, Cascini GL, Tamburrini O (2007) Current status of PET/CT for tumour volume definition in radiotherapy treatment planning for non-small cell lung cancer (NSCLC). Lung cancer 57(2): $125-134$.

6. Hofheinz F, Langner J, Petr J, Beuthien- Baumann B, Steinbach J, et al. (2013) An automatic method for accurate volume delineation of heterogeneous tumors in PET. Medical physics 40(8).

ISSN: 2574-1241

DOI: 10.26717.BJSTR.2019.14.002510

MS Oliveira.Biomed J Sci \& Tech Res

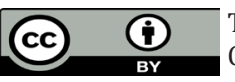

This work is licensed under Creative Commons Attribution 4.0 License

Submission Link: https://biomedres.us/submit-manuscript.php
7. Konert T, Vogel W, MacManus MP, Nestle U, Belderbos J, et al. (2015) PET/CT imaging for target volume delineation in curative intent radiotherapy of non-small cell lung cancer: IAEA consensus report 2014. Radiotherapy and Oncology 116(1): 27-34.

8. Schaefer A, Kremp S, Hellwig D, Rübe C, Kirsch CM, et al. (2008) A contrast-oriented algorithm for FDG-PET-based delineation of tumour volumes for the radiotherapy of lung cancer: derivation from phantom measurements and validation in patient data. European journal of nuclear medicine and molecular imaging 35(11): 1989-1999.

9. Belhassen S, Zaidi H (2010) A novel fuzzy C- means algorithm for unsupervised heterogeneous tumor quantification in PET. Medical physics 37(3): 1309-1324.

10. Hatt M, Le Rest CC, Turzo A, Roux C, Visvikis D (2009) A fuzzy locally adaptive Bayesian segmentation approach for volume determination in PET. IEEE transactions on medical imaging 28(6): 881-893.

11. Sutton MA, Bezdek JC, Cahoon TC (2000) Image segmentation by fuzzy clustering: methods and issues. Handbook of medical imaging: processing and analysis 87-126.

12. Ballangan C, Wang X, Fulham M, Eberl S, Feng D (2011) Lung tumor delineation in PET-CT images using a downhill region growing and a Gaussian mixture model. In Image Processing (ICIP) 2011 18th IEEE International Conference on pp. 2173-2176.

13. Li H, Thorstad WL, Biehl KJ, Laforest R, Su Y, et al. (2008) A novel PET tumor delineation method based on adaptive region- growing and dualfront active contours. Medical physics 35(8): 3711-3721.

14. Onoma DP, Ruan S, Thureau S, Nkhali L, Modzelewski R, et al. (2014) Segmentation of heterogeneous or small FDG PET positive tissue based on a 3D-locally adaptive random walk algorithm. Computerized Medical Imaging and Graphics 38(8): 753-763.

15. Fedorov A, Beichel R, Kalpathy-Cramer J, Finet J, Fillion-Robin JC, et al. (2012) 3D Slicer as an image computing platform for the Quantitative Imaging Network. Magnetic resonance imaging, 30(9): 1323-1341.

16. Vezhnevets V, Konouchine V (2005) Grow Cut: Interactive multi-label ND image segmentation by cellular automata. In proc. of Graphicon 1(4): pp. 150-156.

17. Zhu L, Kolesov I, Gao Y, Kikinis R, Tannenbaum A (2014) An effective interactive medical image segmentation method using fast growcut. In MICCAI workshop on interactive medical image computing.

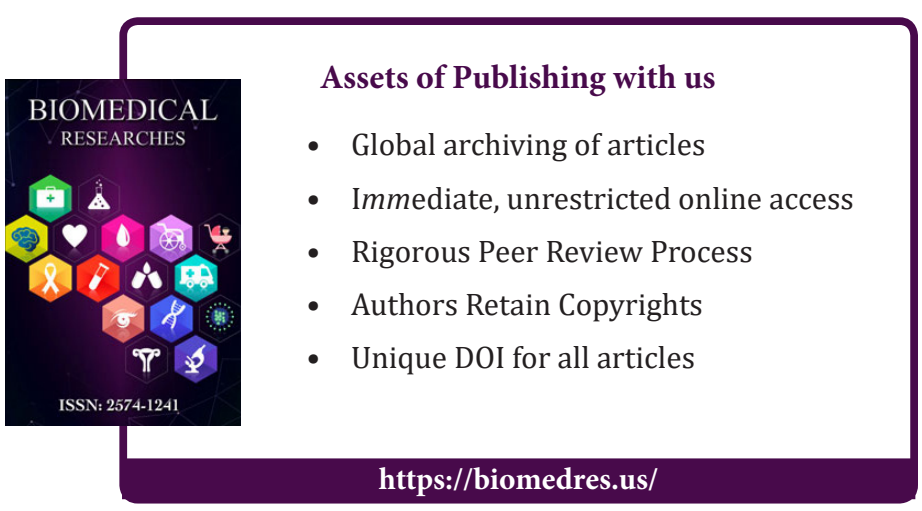

